\title{
Effects of a six-week endurance weight training program on bioelectrical activity of muscles and functional tests in patients with type 2 diabetes mellitus
}

\section{ABSTRACT}

Background. Type 2 diabetes mellitus (T2DM) is a common metabolic disease associated with pathophysiological changes in the neuromuscular system. The present study aimed to investigate the effects of a 6-week endurance training program on muscle strength, electrophysiological parameters, and functional activities in T2DM patients.

Methods. The study period was divided into 6 weeks without training and 6 weeks with training. Twelve T2DM patients participated in this study. During the training period, the same T2DM patients went through an endurance training program. The assessment process included measurement of muscle strength, surface electromyography (SEMG) recording, and functional tests. Twelve healthy individuals were evaluated to compare their data with the T2DM patients.

Results. The results showed that the muscle strength and the amplitudes of the SEMG signals of T2DM patients were lower than those of the healthy subjects. The bioelectric activity of T2DM patients increased after six weeks of the endurance training program. The functional tests showed significant improvement after

Address for correspondence:

Farid Bahrpeyma

Department of Physical Therapy,

Faculty of Medical Sciences,

Tarbiat Modares University, Tehran, Iran

e-mail: bahrpeyf@modares.ac.ir

Clinical Diabetology 2021, 10; 3: 245-251

DOI: $10.5603 /$ DK.a2021.0006

Received: 04.09.2020

Accepted: 28.12 .2020 the endurance training program in T2DM patients, while no significant difference was observed between the T2DM and healthy subjects.

Conclusions. The short-term endurance-training program for lower limb muscles increased muscle strength and SEMG amplitudes of the knee extensor and flexor muscles and improved functional tests in T2DM patients, which may be attributed to neural adaptation after the endurance-training program. (Clin Diabetol 2021; 10; 3: 245-251)

Key words: type 2 diabetes mellitus, endurance training, muscle strength, electromyography, functional tests

\section{Introduction}

Type two diabetes mellitus (T2DM) is a common metabolic disorder, characterized by hyperglycemia due to insulin resistance. T2DM may interfere with the functioning and metabolism of muscles [1]. Hyperglycemia caused by T2DM results in changes in the muscle fiber composition, reduced capillary density, mitochondria dysfunction, and impaired fat metabolism in the muscular tissue [2]. Reduced muscle strength and muscle quality in T2DM may have detrimental effects on mobility, physical activities, and quality of life [1].

Decreased force-generating ability in T2DM can arise from the impairment of the nervous system and/ or intrinsic muscular dysfunctions [3]. Central drives to alpha motoneurons characterize the muscle activation pattern. In addition, the number and type of the acti- 
vated motor units are decisive factors in muscles force production [4]. Surface electromyography (SEMG), as a noninvasive procedure, is an important tool for recording muscular activity. This method provides an estimation of the activity of the neuromuscular system [5].

Numerous exercise training programs may enhance the performance of the neuromuscular system and increase the force-generation capacity in type 2 diabetic patients $[3,6,7]$. Previous studies have proposed different training programs for T2DM, which vary in type, duration, and severity of exercises. The adaptations observed after a training program can depend on its duration. Long-term training programs provide structural adaptations, while short-term training programs are mainly related to neural adaptations [8]. Several studies have demonstrated the effects of 8 to 16 weeks of training on the metabolic properties and muscle strength in T2DM. Botton et al. showed that a 12-week strength training program increased knee extensor and quadriceps muscle thickness in T2DM [6]. Bazzucchi et al. [7] stated that resultant torques and neuromuscular properties of knee extensor muscles in T2DM were improved after 16 weeks of combined strength and endurance training. However, the pattern of adaptation after a short-term training program in T2DM patients with neuromuscular impairments is not well documented.

We propose that T2DM patients with neuromuscular impairment may represent a different pattern of adaptation after training programs. The majority of previous studies have focused on metabolic properties and muscle strength after long-term training programs in T2DM. Hence, we aimed to clarify the effects of a short-term training program on bioelectric activity of the muscles, their force-generating capacity, and functional mobility in T2DM patients.

\section{Methods}

The study was conducted based on a self-controlled design in two separate periods. The first period included a six-week period without training, considered as the control period to eliminate the effects of time. At the second period, participants went through a 6-week training program, characterized as a training period. This means that at first each participant contributed as a control, followed by contributing as an experimental at the second period. The evaluations were performed in three stages, i.e., before the period without any training, before the training period, and after the training period. Moreover, the group of healthy subjects was assessed only once to act as reference data for comparison with the T2DM patients.

\section{Participants}

The T2DM group included 12 patients $(6$ male and 6 female, average age: $51.75 \pm 4.97$, average height: $166.33 \pm 8.37$, average weight: $77.67 \pm 7.51$, average BMI: $28.63 \pm 2.42$ ), while the healthy group included 12 subjects ( 6 male and 6 female, average age: $49.33 \pm 6.56$, average height: $168.33 \pm 10.76$, average weight: $73.08 \pm 11.90$, average BMI: $26.65 \pm 2.23$ ). All participants were informed about the procedures of the study, and they signed a consent form prior to participation. The study was approved by the Ethical Committee of Tarbiat Modares University.

The T2DM was diagnosed for all patients by an endocrinologist. All patients had been suffering from T2DM for 5 to 10 years. They had no history of insulin dependence and just took one or two oral glucoselowering medications [9] with no modification during study period. The glycosylated hemoglobin ( $\mathrm{HbA1C}$ ) values ranged from 7.0 to $9.0 \%$ and fasting plasma glucose concentrations were between 150 to $200 \mathrm{mg}$ per deciliter. The subjects had no history of retinopathy, nephropathy and neuropathy, thyroid and blood pressure diseases and foot ulcer according to endocrinologist examinations. The Michigan Neuropathy Screening test score was less than 6 . None of the participants had a history of neurologic, orthopedic, cardiopulmonary, or rheumatologic diseases, or a history of surgery in the lower limbs. There was no history of middle ear diseases in any of the subjects. The ophthalmoscopy examinations showed that no participants had visual disorders. No regular exercise and physical activity had been performed by the participants during the last two years. The participants who were unwilling or unable to continue with the training program and the testing procedure were excluded from the study. Presence of autonomic neuropathy symptoms during training or testing would result in the exclusion of the subjects from the study as well.

\section{Training program}

The knee extensor and flexor muscles were trained. Prior to training, the one-repetition maximum (1RM) of knee flexion and extension was determined for all participants. The training program was implemented in three sets according to Reverse Pyramid Exercises protocol. The first set included high load (65\% 1RM) and low repetition (25 repetitions), the second set included $60 \% 1 \mathrm{RM}$ and 30 repetition, and the last set included low loads (55\% 1RM) and high repetitions (35 repetitions). Between each set of exercises, the participants had 120 seconds of rest. The difficulty of the exercises was set between 12 and 15 in Borg Scale. 
Heartrate was monitored during the training program using a heartrate monitor (Beurer PM 70). The target heartrate according to the Borg Scale and the American Diabetes Association was set at $65-70 \%$ of the maximal heartrate. The maximum heartrate was calculated as 220 minus the age of the individual. The warm-up and cool-down phases included 5 minutes of cycling with zero load and three repetitions of stretching exercises for hamstring, quadriceps, cuff, and pretibial muscles. Every training sessions lasted nearly $45-50$ minutes. All participants were training 3 days per weeks for 6 weeks. The training program was done under the supervision of an exercise specialist. The blood glucose level has been measured before, in the middle and immediately after the training sessions to determine the probability of hypoglycemia in all participants.

\section{Strength assessment}

To assess muscle strength, the one repetition maximum (1RM) was used. The 1RM is the maximal amount of resistance which can be moved through a full range of motion. In the present study, the 1RM of knee flexion and extension was assessed at all three evaluation sets with hamstring curled and knee extensor machines.

\section{Electromyography assessment}

The bioelectric activity of vastus medialis, vastus lateralis, biceps femoris, and medial hamstring muscles were recorded using SEMG. The SEMG signals were recorded by a 16-channel wireless SEMG device (Fanavaran Technology Company, Iran, 2015). The location of electrode attachment was shaved and cleaned by alcohol. To record the signals of each muscle, a pair of $\mathrm{Ag}-\mathrm{AgCl}$ electrodes (Skintact F-301 Pediatric Electrode) with a center to center distance of $20 \mathrm{~mm}$ were attached to the muscles. The electrodes were placed according to SENIAM recommendation [5]. The ground electrode was placed at the styloid process of the ulna in the non-dominant hand.

The recording was done at $0^{\circ}, 45^{\circ}$, and $90^{\circ}$ flexion during maximal isometric contraction. The knee flexion was done at prone position with a hamstring curl machine, while the knee extension was done at the sitting position with a knee extensor machine. The contraction of the muscles was hold for 10 second, and repeated 3 times. After each repetition, 120 seconds of rest were considered to prevent fatigue.

In order to analyze the collected data, the LabVIEW software application (v14.0, National Instruments, Austin, TX, USA) was used. The sampling frequency of raw SEMG signals was $2000 \mathrm{~Hz}$, and the high and low pass filters were set on 20 and $500 \mathrm{~Hz}$, respectively. The root mean square (RMS) values of the SEMG signals of the knee flexor and extensor muscles during isometric contraction were calculated, and the average of three repetitions was considered as the SEMG amplitude. If the differences of RMSs during each session of contraction were more than $10 \%$ of max RMS, the test was repeated again. The RMS values were normalized to isometric MVC values at 0 degree flexion.

\section{Functional tests}

Two functional tests were used to assess the functional status of patients at three testing sessions. The 6 min walk test (6-MWT) is a functional test that measures the distance covered in 6 minutes without running. A difference of more than 20 meters was considered as a significant change [10]. The Time Up and Go Test (TUG) is a mobility test that measures the time it takes for the subject to get up from a chair, walk three meters forward, turn around, walk back, and sit on the chair [11].

\section{Data analysis}

The data collected in the study were analyzed using SPSS software application (V15.0, IBM Corporation, Armonk, NY, USA). The significance level was considered as $P<0.05$. To analyze the variance of the three testing sessions, the repeated measures ANOVA was used. The Least Significant Difference (LSD) test was used to determine the difference between the sessions. Moreover, independent t-test was used to compare the T2DM patients and the healthy group.

\section{Results}

The Kolmogorov-Smirnov test indicated the normal distribution of the data. The results showed that the RMS of knee flexor and extensor muscles in T2DM was significantly lower than those of the healthy individuals (Tables 1-4).

The results of $1 \mathrm{RM}$ assessment and functional tests are presented in Table 3. The LSD analysis showed the $1 \mathrm{RM}$ of knee extensor and flexor muscles and functional tests at the third stage of assessment was significantly different from the first and second stages of assessment (Tables 5, 6).

The results showed that the $1 \mathrm{RM}$ of knee extensor and knee flexor in healthy subjects was higher than that of the T2DM subjects. After six weeks of training, the $1 \mathrm{RM}$ of knee extensor increased to $41.5 \%$ and that of the knee flexor increased to $31.4 \%$. The $6 \mathrm{MW}$ and TUG test in T2DM patients and healthy subjects showed no significant differences with healthy subjects. The six weeks (non-training) of the control period led to no significant differences; however, after six weeks of training, the $6 \mathrm{MW}$ test increased (10.9\%) and the TUG decreased $(8.8 \%)$ in the T2DM subjects. 
Table 1. The mean values of RMS of knee flexor muscles

\begin{tabular}{lccccc}
\hline Variable & ROM (degree) & D1 (M \pm SD) & D2 (M \pm SD) & D3 (M \pm SD) \\
\hline RMS MH & 90 & $100 \pm 0$ & $98.17 \pm 18.89$ & $104.61 \pm 16.27$ & $100 \pm 0$ \\
& 45 & $105.89 \pm 13.37$ & $109.28 \pm 14.43$ & $117.90 \pm 14.58$ \\
& 0 & $124.53 \pm 14.85$ & $118.23 \pm 17.07$ & $134 \pm 21.4$ & $167.78 \pm 31.78$ \\
RMS BF & 90 & $100 \pm 0$ & $102.67 \pm 9.36$ & $108.13 \pm 13.61$ & $94.63 \pm 13.15$ \\
& 45 & $89.29 \pm 15.99$ & $90.53 \pm 14.11$ & $87.41 \pm 12.55$ \\
\hline
\end{tabular}

BF — biceps femoris; D1 - T2DM participant first stage assessment; D2 - T2DM participant second stage assessment; D3 — T2DM participant third stage assessment; $\mathrm{H}$ - healthy participant assessment; $\mathrm{M} \pm \mathrm{SD}$ - mean \pm standards deviation; $\mathrm{MH}$ - medial hamstring; $\mathrm{RMS}$ - root mean square; $\mathrm{ROM}$ range of motion

Table 2. The comparison of RMS of knee flexor muscles between three assessment stages and healthy subjects

\begin{tabular}{|c|c|c|c|c|c|c|c|c|}
\hline \multirow[t]{2}{*}{ Variable } & \multirow[t]{2}{*}{ ROM (degree) } & \multirow[t]{2}{*}{ ANOVA } & \multicolumn{3}{|c|}{ LSD } & \multicolumn{3}{|c|}{ t-test } \\
\hline & & & D1-D2 & D1-D3 & D2-D3 & D1 - H & D2 - H & D3 - H \\
\hline \multirow[t]{3}{*}{$\mathrm{MH}$} & 90 & 0.33 & - & - & - & $\mathrm{NaN}$ & 0.74 & 0.34 \\
\hline & 45 & $<0.01 *$ & - & Sig* & Sig* & $<0.01 *$ & $<0.01 *$ & $0.01 *$ \\
\hline & 0 & $<0.01 *$ & - & Sig* & Sig* & $<0.01 *$ & $<0.01 *$ & $<0.01 *$ \\
\hline \multirow[t]{3}{*}{$\mathrm{BF}$} & 90 & 0.07 & - & - & - & $\mathrm{NaN}$ & 0.34 & 0.06 \\
\hline & 45 & 0.18 & - & - & - & 0.44 & 0.71 & 0.73 \\
\hline & 0 & $0.04^{*}$ & - & - & $\mathrm{Sig}^{*}$ & 0.25 & 0.08 & 0.80 \\
\hline
\end{tabular}

BF - biceps femoris; D1 - T2DM participant first stage assessment; D2 - T2DM participant second stage assessment; D3 - T2DM participant third stage assessment; $\mathrm{H}$ - healthy participant assessment; LSD — least significant difference; $\mathrm{MH}$ - medial hamstring; NaN — not a number; RMS — root mean square; ROM — range of motion; Sig — significant

Table 3. The mean values of RMS of knee extensor muscles

\begin{tabular}{lccccc}
\hline Variable & ROM (degree) & D1 (M \pm SD) & D2 (M \pm SD) & D3 (M \pm SD) \\
\hline RMS VMO & 90 & $149.26 \pm 27.11$ & $149.80 \pm 32.71$ & $157.03 \pm 30.32$ & $226.28 \pm 45.82$ \\
& 45 & $129.34 \pm 29.91$ & $133.10 \pm 23.35$ & $145.38 \pm 29.78$ & $204.39 \pm 27.07$ \\
RMS VL & 0 & $100 \pm 0$ & $101.08 \pm 9.25$ & $107.39 \pm 10.23$ & $100 \pm 0$ \\
& 90 & $164.75 \pm 23.25$ & $166.49 \pm 22.15$ & $183.02 \pm 29.30$ & $239.37 \pm 35.85$ \\
& 45 & $135.66 \pm 19.60$ & $142.40 \pm 23.12$ & $156 \pm 24.58$ & $213.56 \pm 41.21$ \\
\hline
\end{tabular}

D1 - T2DM participant first stage assessment; D2 - T2DM participant second stage assessment; D3 - T2DM participant third stage assessment; $\mathrm{H}$ - healthy participant assessment; $\mathrm{M} \pm \mathrm{SD}$ - mean \pm standards deviation; $\mathrm{RMS}$ - root mean square; $\mathrm{ROM}$ - range of motion; $\mathrm{VL}$ - vastus lateralis; VMO - vastus medialis oblique

\section{Discussion}

The results of the present study showed that the muscle strength and RMS of knee extensor and flexor muscles in healthy individuals were significantly higher than those of the T2DM patients. The six-week endurance training program increased the muscle strength and the RMS of knee extensor and flexor muscles in T2DM patients. The functional tests assessment showed that there were no significant differences between T2DM and healthy subjects.

Numerous studies have reported that the muscle strength of diabetic patients decreased as a result of the complications of the disease compared to healthy subjects [12-15]. Similarly, the present study indicated that $1 \mathrm{RM}$ and bioelectrical activation of knee flexor and extensor muscles in T2DM were lower than those of the healthy subjects prior to the appearance of neuropathological symptoms. Hatef et al. [15] showed that the maximal peak torques and RMS of knee extensor and flexor muscles in healthy individuals were higher than those of the T2DM patients, which was attributed to the duration of the disease and the appearance of neuropathological symptoms [15]. A linear relationship was observed between the increase in RMS and isometric 
Table 4. The comparison of RMS of knee extensor muscles between three assessment stages and healthy subjects

\begin{tabular}{|c|c|c|c|c|c|c|c|c|}
\hline \multirow[t]{2}{*}{ Variable } & \multirow[t]{2}{*}{ ROM (degree) } & \multirow[t]{2}{*}{ ANOVA } & \multicolumn{3}{|c|}{ LSD } & \multicolumn{3}{|c|}{ T-test } \\
\hline & & & D1-D2 & D1-D3 & D2-D3 & D1-H & D2-H & D3-H \\
\hline \multirow[t]{3}{*}{ VMO } & 90 & 0.20 & - & - & - & $<0.01 *$ & $<0.01 *$ & $<0.01 *$ \\
\hline & 45 & $0.03^{*}$ & - & Sig* & - & $<0.01 *$ & $<0.01 *$ & $<0.01 *$ \\
\hline & 0 & $0.01 *$ & - & Sig* & Sig* $^{*}$ & $\mathrm{NaN}$ & 0.69 & $0.04^{*}$ \\
\hline \multirow[t]{3}{*}{ VL } & 90 & $<0.01^{*}$ & - & Sig* & Sig* $^{*}$ & $<0.01 *$ & $<0.01^{*}$ & $<0.01 *$ \\
\hline & 45 & $<0.01 *$ & - & Sig* & $\mathrm{Sig}^{*}$ & $<0.01 *$ & $<0.01^{*}$ & $<0.01 *$ \\
\hline & 0 & $<0.01^{*}$ & Sig* & Sig* & Sig* & $\mathrm{NaN}$ & $<0.01^{*}$ & $<0.01^{*}$ \\
\hline
\end{tabular}

D1 - T2DM participant first stage assessment; D2 - T2DM participant second stage assessment; D3 - T2DM participant third stage assessment; $\mathrm{H}$ - healthy participant assessment; LSD — least significant difference; NaN — not a number; RMS — root mean square; ROM — range of motion; Sig significant; VL — vastus lateralis; VMO — vastus medialis oblique

Table 5. The mean values of knee extensor and flexor strength and functional tests

\begin{tabular}{|c|c|c|c|c|}
\hline Variable & $\mathrm{D} 1(\mathrm{M} \pm \mathrm{SD})$ & $\mathrm{D} 2(\mathrm{M} \pm \mathrm{SD})$ & $\mathrm{D} 3(\mathrm{M} \pm \mathrm{SD})$ & $H(M \pm S D)$ \\
\hline TUG & $6.33 \pm 0.88$ & $6.28 \pm 0.85$ & $5.73 \pm 0.68$ & $6.03 \pm 0.75$ \\
\hline $6 \mathrm{MW}$ & $514.17 \pm 64.66$ & $519.75 \pm 65.69$ & $576.42 \pm 61.89$ & $55.9 \pm 83.23$ \\
\hline 1RM EXT & $27.33 \pm 9.06$ & $29 \pm 9.29$ & $40.75 \pm 12.17$ & $51.5 \pm 12.66$ \\
\hline 1RM FLX & $15.58 \pm 7.22$ & $18.33 \pm 7.58$ & $24.08 \pm 11.18$ & $28.25 \pm 13.71$ \\
\hline
\end{tabular}

1RM - one-repetition maximum; 6MW - six mean walk test; D1 - T2DM participant first stage assessment; D2 - T2DM participant second stage assessment; D3 - T2DM participant third stage assessment; Ext - extensor; Flex — flexor; $\mathrm{H}$ - healthy participant assessment; TUG — time up and go test

Table 6. The comparison of knee extensor and flexor strength and functional test between three assessment stages and healthy subjects

\begin{tabular}{|c|c|c|c|c|c|c|c|}
\hline \multirow[t]{2}{*}{ Variable } & \multirow[t]{2}{*}{ ANOVA } & \multicolumn{3}{|c|}{ LSD } & \multicolumn{3}{|c|}{ t-test } \\
\hline & & D1-D2 & D1-D3 & D2-D3 & D1 - H & $\mathrm{D} 2-\mathrm{H}$ & D3 - H \\
\hline TUG & $<0.01 *$ & - & Sig* & Sig* & 0.50 & 0.21 & 0.20 \\
\hline $6 \mathrm{MW}$ & $<0.01^{*}$ & - & Sig* & Sig* & 0.15 & 0.20 & 0.57 \\
\hline 1RM EXT & $<0.01^{*}$ & - & Sig* & Sig* & $<0.01 *$ & $<0.01 *$ & 0.20 \\
\hline 1RM FLX & $<0.01^{*}$ & - & Sig* & Sig* & 0.01 * & $0.03^{*}$ & 0.42 \\
\hline
\end{tabular}

1RM - one-repetition maximum; $6 \mathrm{MW}$ - six mean walk test; D1 - T2DM participant first stage assessment; D2 - T2DM participant second stage assessment; D3 - T2DM participant third stage assessment; Ext - extensor; Flex — flexor; $\mathrm{H}$ - healthy participant assessment; LSD — least significant difference; TUG - time up and go test

muscle strength [16]. The complications of T2DM lead to neuromuscular dysfunction, resulting in decreased forcegenerating ability in relation to the decreased bioelectric activity of muscles in T2DM patients [17].

Hatef et al. [15] studied the neuromuscular properties of muscles after a fatigue protocol [15], while the present study assessed the effects of a short-term training program on neuromuscular properties of knee flexor and extensor muscles. The results showed that muscle force and the amplitude of the bioelectrical activity of knee extensor and flexor muscles increased after six weeks of endurance training. A similar study indicated that the muscle strength and efficiency of the neuromuscular system increased at the early stages of strength training without obvious changes in the amplitude of SEMG signals [18]. Increased force generation at the early stages of training can indicate that the role of neural adaptation is greater than that of the structural adaptation of muscles. Neural adaptations include some changes in the coordination of muscles and learning movements in the neuromuscular system, improving the neuromuscular activity of the muscles during force generation [8].

The diabetes-related international organizations recommend multiple exercise training programs in T2DM patients as non-pharmacological therapeutic strategy. Numerous exercises training including endurance, strength and combined training programs with different intensity and duration have brought beneficial effects about biochemical and physical properties in 
T2DM [19]. The strength training programs compared to endurance training are introduced more recently in T2DM patients which improves muscle strength and motor functions [20]. But the exact effects of various type, intensity and duration of strength training has not been elucidated yet in T2DM patients. A review study evaluated the effects of resistance training on T2DM patients. The results showed that resistance training increased the muscle strength of T2DM patients in the lower limb muscles more than the upper limb muscles [21]. In addition, Herriott et al. [22] and Egger et al. [23] indicated that resistance training increased muscle strength in T2DM patients. The type, intensity and duration of training in the above-mentioned studies are variable. The duration of the training varied between 8 and 16 weeks and the training protocols involved the whole body training with high loads and were not specific to particular muscles. Long term and high intensity strength training programs may induce high blood pressure and muscle injuries in T2DM patients [24, 25]. In contrast to previous studies, we found that a short-term endurance weight training program for knee flexor and extensor muscles could affect muscle strength and the neuromuscular properties of the muscles. The relationship between muscle strength and RMS indicated that the bioelectric activity of knee flexor and extensor muscles increased in parallel with $1 \mathrm{RM}$ enhancement after a short-term training program, which could be attributed to the neural adaptation of the training [26].

Improving functional activity in T2DM patients is one of the most important goals of therapeutic exercise [27]. In addition to impaired muscle strength in T2DM patients, the T2DM patients are exposed to decreased functional capacity, physical disability, and impaired functional mobility [3]. Decreased functional capacity of movement can be affected by impaired intrinsic properties of muscles, including decreased muscle mass, impaired metabolism, and dysfunction of mitochondria [28]. Moreover, diabetic peripheral neuropathy may affect the functional ability of movement [29].

The TUG and 6MW tests are used to measure functional activities [30]. The results from the present study showed improvement of functional tests after the training program, which means that the distance increased in the $6 \mathrm{MW}$ test and the time duration decreased in the TUG test. Hulens et al. [31] demonstrated that individuals with higher strength in the quadriceps muscle had higher scores in the $6 \mathrm{MW}$ test. We proposed that exercise training to strengthen the knee extensor muscles could improve walking ability in T2DM patients. In the present study, the training program was designed for knee extensor and flexor muscles; hence, the improvement in the $6 \mathrm{MW}$ test can be attributed to increased knee flexor and extensor strength in T2DM patients. Similarly, following a 12week strength and endurance training program, Allet et al. [32] reported improvement in walking speed, balance, muscle strength, joint mobility, and functional tests in T2DM patients. Another study by ljzerman et al. [13] showed that the $6 \mathrm{MW}$ test performance in T2DM patients with and without DPN was significantly lower than healthy individuals; however, the TUG test was only significant between the T2DM patients with neuropathy and healthy individuals. Nonetheless, their results are controversial since the healthy individuals and T2DM patients were not completely matched in terms of height, weight, and BMI. Also, the functional tests are affected by age, height, BMI, sex, muscle strength and endurance, and weight [33].

Mueller et al. [34] reported that weight-bearing and non-weight-bearing exercises led to improvement in the $6 \mathrm{MW}$ test; however, the effects of weight-bearing exercises were greater. A recent review study by Hwang et al. showed that all types of training, including endurance, resistance, balance, and combined training had beneficial effects on the 6MW and TUG tests in type 2 diabetic patients [35]. The results of the present study show that the short-term endurance weight training for knee extensor and flexor muscles can lead to increased muscle strength and increased capacity for functional activities in T2DM patients, which may result in an independent lifestyle.

\section{Conclusion}

The T2DM patients have reduced muscle forcegenerating ability and functional activity compared to healthy subjects. Consequently, a short-term endurance training program for T2DM patients increases muscle strength and muscle bioelectric activity, while it can improve functional tests, which may be related to neural adaptation at the early stages of training.

\section{Acknowledgments}

This study was a residency thesis and was supported by Tarbiat Modares University. The authors would like to thank the subjects for their participation in this study.

\section{Conflicts of interest}

The authors declare no conflict of interest regarding publication of this manuscript.

\section{REFERENCES}

1. Bianchi L, Volpato S. Muscle dysfunction in type 2 diabetes: a major threat to patient's mobility and independence. Acta Diabetol. 2016; 53(6): 879-889, doi: 10.1007/s00592-016-0880-y, indexed in Pubmed: 27393005. 
2. Phielix E, Mensink M. Type 2 diabetes mellitus and skeletal muscle metabolic function. Physiol Behav. 2008; 94(2): 252-258, doi: 10.1016/j.physbeh.2008.01.020, indexed in Pubmed: 18342897.

3. Orlando G, Balducci S, Bazzucchi I, et al. Neuromuscular dysfunction in type 2 diabetes: underlying mechanisms and effect of resistance training. Diabetes Metab Res Rev. 2016; 32(1): 40-50, doi: 10.1002/dmrr.2658, indexed in Pubmed: 25950170.

4. Contessa P, De Luca CJ. Neural control of muscle force: indications from a simulation model. J Neurophysiol. 2013; 109(6): 1548-1570, doi: 10.1152/jn.00237.2012, indexed in Pubmed: 23236008.

5. Merlo A, Campanini I. Technical aspects of surface electromyography for clinicians. The Open Rehabilitation Journal. 2010; 3(1): 98-109, doi: 10.2174/1874943701003010098.

6. Botton CE, Umpierre D, Rech A, et al. Effects of resistance training on neuromuscular parameters in elderly with type 2 diabetes mellitus: A randomized clinical trial. Exp Gerontol. 2018; 113: 141-149, doi: 10.1016/j.exger.2018.10.001, indexed in Pubmed: 30296453.

7. Bazzucchi I, De Vito G, Felici F, et al. Effect of exercise training on neuromuscular function of elbow flexors and knee extensors of type 2 diabetic patients. J Electromyogr Kinesiol. 2015; 25(5): 815-823, doi: 10.1016/j.jelekin.2015.06.008, indexed in Pubmed: 26194594

8. Folland JP, Williams AG. The adaptations to strength training : morphological and neurological contributions to increased strength. Sports Med. 2007; 37(2): 145-168, doi: 10.2165/00007256200737020-00004, indexed in Pubmed: 17241104.

9. Preiser JC, Provenzano B, Mongkolpun W, et al. Perioperative management of oral glucose-lowering drugs in the patient with type 2 diabetes. Anesthesiology. 2020; 133(2): 430-438, doi: 10.1097/ ALN.0000000000003237, indexed in Pubmed: 32667156.

10. Kervio G, Carre F, Ville NS. Reliability and intensity of the six-minute walk test in healthy elderly subjects. Med Sci Sports Exerc. 2003; 35(1): 169-174, doi: 10.1097/00005768-200301000-00025, indexed in Pubmed: 12544651.

11. Bohannon RW. Reference values for the timed up and go test: a descriptive meta-analysis. J Geriatr Phys Ther. 2006; 29(2): 64-68, doi: 10.1519/00139143-200608000-00004, indexed in Pubmed: 16914068.

12. Andersen $H$, Nielsen $S$, Mogensen $C E$, et al. Muscle strength in type 2 diabetes. Diabetes. 2004; 53(6): 1543-1548, doi: 10.2337/ diabetes.53.6.1543, indexed in Pubmed: 15161759.

13. IJzerman TH, Schaper NC, Melai T, et al. Lower extremity muscle strength is reduced in people with type 2 diabetes, with and without polyneuropathy, and is associated with impaired mobility and reduced quality of life. Diabetes Res Clin Pract. 2012; 95(3): 345-351, doi: 10.1016/j.diabres.2011.10.026, indexed in Pubmed: 22104262

14. Almurdhi MM, Reeves ND, Bowling FL, et al. Reduced lower-limb muscle strength and volume in patients with type 2 diabetes in relation to neuropathy, intramuscular fat, and vitamin d levels. Diabetes Care. 2016; 39(3): 441-447, doi: 10.2337/dc15-0995, indexed in Pubmed: 26740641.

15. Hatef B, Bahrpeyma F, Mohajeri Tehrani MR. The comparison of muscle strength and short-term endurance in the different periods of type 2 diabetes. J Diabetes Metab Disord. 2014; 13(1): 22, doi: 10.1186/2251-6581-13-22, indexed in Pubmed: 24476108.

16. Luera MJ, Stock MS, Chappell ADW. Electromyographic amplitude vs. concentric and eccentric squat force relationships for monoarticular and biarticular thigh muscles. J Strength Cond Res. 2014; 28(2): 328-338, doi: 10.1519/JSC.0b013e3182a1f434, indexed in Pubmed: 23897014.

17. Askary-Ashtiani A, Ghanjal A, Motaqi M, et al. The isokinetic and electromyographic assessment of knee muscles strength in the short- and long-term type 2 diabetes. Asian J Sports Med. 2016; 7(4): e37008, doi: 10.5812/asjsm.37008, indexed in Pubmed: 28144413.

18. Shahrjerdi S, Bahrpeyma F, Savelberg HH, et al. Effect of a 6-week strength-training program on neuromuscular efficiency in type 2 diabetes mellitus patients. Diabetol Int. 2020; 11(4): 376-382, doi: 10.1007/s13340-020-00432-y, indexed in Pubmed: 33088645.

19. Colberg SR, Sigal RJ, Yardley JE, et al. Exercise and type 2 diabetes: American College of Sports Medicine and the American Diabetes
Association: joint position statement. Exercise and type 2 diabetes. Med Sci Sports Exerc. 2010; 42(12): 2282-2303, doi: 10.1249/ MSS.0b013e3181eeb61c, indexed in Pubmed: 21084931.

20. Lee J, Kim D, Kim C. Resistance training for glycemic control, muscular strength, and lean body mass in old type 2 diabetic patients: a meta-analysis. Diabetes Ther. 2017; 8(3): 459-473, doi: 10.1007/s13300-017-0258-3, indexed in Pubmed: 28382531.

21. Hovanec N, Sawant A, Overend TJ, et al. Resistance training and older adults with type 2 diabetes mellitus: strength of the evidence. J Aging Res. 2012; 2012: 284635, doi: 10.1155/2012/284635, indexed in Pubmed: 22988507.

22. Herriott MT, Colberg SR, Parson HK, et al. Effects of 8 weeks of flexibility and resistance training in older adults with type 2 diabetes. Diabetes Care. 2004; 27(12): 2988-2989, doi: 10.2337/ diacare.27.12.2988, indexed in Pubmed: 15562222.

23. Egger A, Niederseer D, Diem G, et al. Different types of resistance training in type 2 diabetes mellitus: effects on glycaemic control, muscle mass and strength. Eur J Prev Cardiol. 2013; 20(6): 1051-1060, doi: 10.1177/2047487312450132, indexed in Pubmed: 22637738.

24. Hovanec N, Sawant A, Overend TJ, et al. Resistance training and older adults with type 2 diabetes mellitus: strength of the evidence. J Aging Res. 2012; 2012: 284635, doi: 10.1155/2012/284635, indexed in Pubmed: 22988507.

25. Gordon PL, Vannier E, Hamada K, et al. Resistance training alters cytokine gene expression in skeletal muscle of adults with type 2 diabetes. Int J Immunopathol Pharmacol. 2006; 19(4): 739-749, doi: 10.1177/039463200601900404, indexed in Pubmed: 17166396.

26. Cadore EL, Pinto RS, Pinto SS, et al. Effects of strength, endurance, and concurrent training on aerobic power and dynamic neuromuscular economy in elderly men. J Strength Cond Res. 2011; 25(3): 758-766, doi: 10.1519/JSC.0b013e318207ed66, indexed in Pubmed: 21311345.

27. Volpato S, Bianchi L, Lauretani F, et al. Role of muscle mass and muscle quality in the association between diabetes and gait speed. Diabetes Care. 2012; 35(8): 1672-1679, doi: 10.2337/ dc11-2202, indexed in Pubmed: 22596176.

28. Kono K, Nishida Y, Moriyama Y, et al. Investigation of factors affecting the six-minute walk test results in hemodialysis patients. Ther Apher Dial. 2014; 18(6): 623-627, doi: 10.1111/17449987.12177, indexed in Pubmed: 24674327.

29. Frier B, Yang P, Taylor A. Diabetes, aging and physical activity. European Review of Aging and Physical Activity. 2006; 3(2): 63-73, doi: 10.1007/s11556-006-0010-x.

30. van Sloten TT, Savelberg HH, Duimel-Peeters IGP, et al. Peripheral neuropathy, decreased muscle strength and obesity are strongly associated with walking in persons with type 2 diabetes without manifest mobility limitations. Diabetes Res Clin Pract. 2011; 91(1): 32-39, doi: 10.1016/j.diabres.2010.09.030, indexed in Pubmed: 20965601.

31. Hulens M, Vansant G, Claessens AL, et al. Predictors of 6-minute walk test results in lean, obese and morbidly obese women. Scand J Med Sci Sports. 2003; 13(2): 98-105, doi: 10.1034/j.16000838.2003.10273.x, indexed in Pubmed: 12641641.

32. Allet L, Armand S, De Bie RA et al. The gait and balance of patients with diabetes can be improved: a randomised controlled trial. Diabetologia. 2010; 53(3): 458-66.

33. Barry E, Galvin R, Keogh C, et al. Is the timed up and go test a useful predictor of risk of falls in community dwelling older adults: a systematic review and meta-analysis. BMC Geriatr. 2014; 14: 14, doi: 10.1186/1471-2318-14-14, indexed in Pubmed: 24484314.

34. Mueller MJ, Tuttle $\amalg$, Lemaster JW, et al. Weight-bearing versus nonweight-bearing exercise for persons with diabetes and peripheral neuropathy: a randomized controlled trial. Arch Phys Med Rehabil. 2013; 94(5): 829-838, doi: 10.1016/j.apmr.2012.12.015, indexed in Pubmed: 23276801.

35. Hwang S, Woo Y. Fall prevention strategies in community-dwelling older adults aged 65 or over with type 2 diabetes mellitus: a systematic review and meta-analysis. Physical Therapy Rehabilitation Science. 2018; 7(4): 197-203, doi: 10.14474/ptrs.2018.7.4.197. 